\title{
Multispecies Interactions in Biofilms and Implications to Safety of Drinking Water Distribution System
}

\author{
Rine Christopher Reuben ${ }^{1,2}$, Pravas Chandra Roy ${ }^{1}$, Shovon Lal Sarkar', Sang-Do Ha ${ }^{3}$, and lqbal Kabir Jahid ${ }^{1 *}$ \\ ${ }^{1}$ Department of Microbiology, Jashore University of Science and Technology, Jashore-7408, Bangladesh \\ ${ }^{2}$ Department of Science Laboratory Technology, Nasarawa State Polytechnic, Lafia, Nigeria \\ ${ }^{3}$ School of Food Science and Technology, Chung-Ang University, Ansung 17546, Republic of Korea
}

Received: July 15, 2019 / Revised: September 6, 2019 / Accepted: September 6, 2019

In the aquatic environment, microorganisms are predominantly organized as biofilms. Biofilms are formed by the aggregation of microbial cells and are surrounded by a matrix of extracellular polymeric substances (EPS) secreted by the microbial cells. Biofilms are attached to various surfaces, such as the living tissues, indwelling medical devices, and piping of the industrial potable water system. Biofilms formed from a single species has been extensively studied. However, there is an increased research focus on multispecies biofilms in recent years. It is important to assess the microbial mechanisms underlying the regulation of multispecies biofilm formation to determine the drinking water microbial composition. These mechanisms contribute to the predominance of the best-adapted species in an aquatic environment. This review focuses on the interactions in the multispecies biofilms, such as coaggregation, co-metabolism, cross-species protection, jamming of quorum sensing, lateral gene transfer, synergism, and antagonism. Further, this review explores the dynamics and the factors favoring biofilm formation and pathogen transmission within the drinking water distribution systems. The understanding of the physiology and biodiversity of microbial species in the biofilm may aid in the development of novel biofilm control and drinking water disinfection processes.

Keywords: Multispecies, biofilm, water, disease, biodiversities

\section{Introduction}

Water is one major and essential need of all humans, as such, it should be readily accessible, safe and adequately supply to all. Unfortunately, high populations of people globally do not have access to purified water sources, thereby constituting a grave sanitation consequence. The impact of poor hygiene and sanitation as a result of the consumption of unsafe water results in an estimated 3.2 percent death globally [1]. It is highly pertinent to note that in most developing countries, the

\section{* Corresponding author}

Tel: +88-01709-818127

E-mail: ikjahid_mb@just.edu.bd

๑ 2019, The Korean Society for Microbiology and Biotechnology majority of people have inadequate access to safe water in spite of the United Nations effort in improving water quality [2]. It is of significant importance also to note that there is a direct relationship between disease burden and water quality. As such, global disease burden can be reduced by approximately 4 percent by improving water quality [1]. Globally, it is suggested that waterborne diseases have an estimated economic cost of nearly $\$ 12$ billion annually while about $\$ 1$ billion is annually spent in the United States alone [3].

In nature, microorganisms rarely exist as planktonic or free-living cells. They are largely found in enclosed matrix, forming complex communities [4]. Despite this, most of the biofilm research has extensively focused on in vitro mono-cultures biofilm formation [5]. Only more 
recently has the focus been moved toward the understanding of microbial diversity and interaction in the natural environment. Furthermore, the increased discoveries of the unique metabolic capabilities of microbial communities have drawn more interest to microbial lifestyle in natural ecosystems [4]. This has unraveled emergent microbial properties not previously detected in free-living cells.

Microorganisms readily attach to diverse surfaces and subsequently form biofilms in aquatic environments. The impact of biofilm formation in medicine, changing antimicrobial therapeutics and comprehension of chronic and emerging infections has been tremendous in recent times. Despite the intensive research on this topic, the impact of biofilm on ecosystems, and more specifically, in the aquatic environment has not been given the same importance. In aquatic ecosystems, biofilms have been recognized as the predominant forms of life due to innumerable benefits bacterial interactions within this matrix provide.

This review describes the various interactions in multispecies biofilms including coaggregation of microbial cells, cross-species interaction, and co-metabolism, lateral gene transfer, jamming of quorum sensing, synergism and antagonism. We also provide updates on the role of biofilms in disease transmission and dynamics, their influence on distribution systems of drinking water, and finally the effects of protozoan grazing on aquatic biofilms.

\section{Biofilms}

Donlan and Costerton [6] defined biofilm as a community of microorganisms adhering to biotic or abiotic surfaces and embedded with a self-produced extracellular polymeric substances (EPS) which facilitate survival in an adverse environment. Biofilms can also be seen as complex architectural assemblies of microorganisms on or in biotic and abiotic surfaces and interfaces, characterized by interactions between populations. They have survived as self-organized, three-dimensional structures that exhibit altered genotypic and phenotypic characteristics [7]. The ubiquity nature of biofilms enables them to thrive and survive in different water systems (including aquatic and industrial), environments and devices (medical) which are of great public health importance
[6]. In nature, about 99 percent of bacteria exist in biofilms while 65 percent of infections in the hospital are attributed to biofilms [8]. When in biofilms, bacteria usually show profound differences in both morphological and physiological features vis-à-vis planktonic cultures. Some of the most profound features of bacteria in biofilms include resistance to unfavorable environmental factors; desiccation, pollutants, antimicrobial agents (in nature), host defenses, and protozoan grazing [9, 10].

The presence of biofilms containing pathogens such as Aeromonas spp, Escherichia coli, and Pseudomonas spp. in drinking water constitute a great potential risk to public health [11]. This is because biofilms can contain a very high number of microbial cells, up to $1.0 \times 10^{9}$ per clump, capable of causing infections when ingested. However, little is known on bacterial survival and potentiality to cause disease in biofilms.

\section{Multispecies Biofilms}

Mixed microbial populations exist in different niches (including aquatic systems), having distinct phenotypic variants which have a significant effect on the overall activities of microbial communities in biofilm [12]. Bacteria attach to such surfaces either as discrete and separate individual cells, aggregates or biofilm communities interacting with other cells thereby forming channels for both nutrients and metabolic by-products circulation which often exists as a three-dimensional architectural structure [13]. Multispecies biofilm can solely comprise of single individual microbial strains or multiple strains within a habitat [8]. The diversity of population reduces the impact of environmental stress because wide range of environmental conditions are extended for the survival of different microbial subpopulations within their community. Different physiological and regulatory alterations occur with multispecies biofilms as a result of microbial interactions. This eventually favors the selection and survival of the most adapted strains or mutants. Consequently, these aid in shaping multispecies biofilm communities as certain species may eventually emerge or disappear.

Recently, we reviewed the roles played by multispecies biofilms with regards to food safety, where we provided detailed interactions and the importance of multispecies biofilms in foods [7]. Multispecies biofilms generally 
show more resistance to disinfectants because they secrete more EPS compared with mono-species biofilms.

\section{Characteristics of Biofilms in Aquatic Environments}

Microbial communities existing as biofilms are rarely planktonic in the aquatic environment. They adhere to different surfaces of living tissues, dead matter or devices which prevent them from external forces capable of sweeping them away [14]. When attached to a surface, Pseudomonas aeruginosa biofilm often produces an exopolysaccharide known as alginate, which serves as an extracellular matrix. Also, V. cholera producing this same exopolymer barrier form biofilms on different surfaces (both biotic and abiotic), which provides protection to the cells. $V$. cholerae also forms a rugose colony in multispecies biofilms isolated from Ganges Delta, Bangladesh [13].

Furthermore, within the aquatic environments, biofilms can be highly diverse depending on the substratum where they develop. In natural environments, biofilms grow upon inert substrata such as sand, sediment, rocks and cobbles; non-living organic substrata such as wood, leaf litter or particular organic matter; and living plants such as aquatic macrophytes and macroalgae [15]. Multispecies biofilms composition, structure, diversity and metabolism are largely determined by the nature and kind of substratum such communities are formed. Furthermore, it has been demonstrated that substratum properties which support biofilms development also determine cells accumulation patterns especially in the early stages of biofilms formation [16]. Table 1 shows the multispecies biofilm growth in different substratum.

Rocks. Biofilms attached to rock surfaces (also to gravel and cobbles) are referred to as epilithic biofilms or epilithon in rivers, marine environments and lakes [17]. Unlike biofilms formed on other substrata, epilithic biofilms have a more complex structure with higher algal biomass and are more independent on seasonal fluctuations [18].

Stones provide a three-dimensional physical habitat for biofilm biomass. The developing biofilms are greatly influenced by rock characteristics such as surface texture, stone size, and stone orientation [19]. On the one hand, a rougher surface can cause greater biofilm accumulation on rocks since it increases the sedimentation efficiency and cell adhesion and further protects the biofilm from disturbances such as scouring and grazing [19]. On the other hand, stone size and stability determine the algal resistance to scour. Regarding stone orientation, horizontal surfaces consistently harbor more biofilm than on vertical surfaces [19]. Biofilms developing on rocks have structural stability and close spatial relationship between bacteria and algae favor the bacterial use of fresh labile organic compounds released by algae, which affect the metabolism of the whole biofilm.

Sediment. Microorganisms attached to sand and gravel (sandy sediments) are referred to as epipsammic, while when developing on muddy sediments (clay or silt) they are known as epipelic [20]. Sediments are usually hard and inert substrata with a smaller size than other substrates. In rivers, biofilms developing on sediment play key role especially in the decomposition of organic matter and are also highly heterotrophic when compared to rock biofilms.

Colonization and microbial community development on sediments depend on the roughness, size and surface area of the grains. Specifically, microbial colonization on

Table 1. Biofilm growth in substratum.

\begin{tabular}{cllc}
\hline S/N & \multicolumn{1}{c}{ Organism (s) } & Substratum of biofilm formation & References \\
\hline 1 & Heterotrophic bacteria, fungi and protozoa & Rocks & 16,21 \\
2 & Cyanobacteria, euglenids and diatoms & Sediments & 21,38 \\
3 & Bacteria, archaea, algae and metazoans & Dead plant Material & 20,23, \\
4 & Bacteria and algae & Living plants & 29,30 \\
5 & Cyanobacteria, bacteria and protozoans & Suspended aggregates & $29-31$ \\
6 & Gram-positive bacteria and diatoms & Man-made surfaces & 34,36 \\
\hline
\end{tabular}


sediment grains is proportional to the grain surface area. However, these assumptions can be applied only from coarse grains to fine silt because clay particles are very rarely colonized since they are too small and smooth. In addition, the colonization of microorganisms is determined by particulate size and roughness [19-21].

In aquatic environments, sediments usually show a profile zonation in depth if they are sufficiently thick. In this sense, sediment thickness creates marked physical and chemical gradients that determine changes in the sediment biofilm community. In this regard, surface sediments support heterotrophic communities that include more opportunistic species. Bacterial density and activity are commonly higher in surface sediments and decrease with depth [9]. In rivers, the sediment hyporheic zone (interface between the river channel and groundwater) offers protection against unfavorable conditions to biofilm communities formed on sediments. Also, grain size, sediment shape, and composition determine the porosity and hydraulic conductivity of the sediment, and influence most physical and chemical processes [21].

Dead plants materials. Dead plant materials (including wood and leaves) biofilm are named epixylic biofilm or epixylon [17]. In this case, the substratum (i.e. dead organic matter surfaces) presents both physical support medium for microbial colonization and organic matter source for microbes. The relevance of the different organisms forming epixylic biofilms is markedly different from other biofilm types. Different phyla of fungi are the principal microbial groups growing on dead plant material, mainly as a result of their lignocellulose degrading ability. However, bacteria, archaea, algae and metazoans also form this kind of biofilms [22].

Different intrinsic and extrinsic factors influence the growth of microorganisms on dead plants materials. Intrinsic factors refer to dead organic matter quality, such as cuticle toughness, nutrient content (particularly nitrogen and phosphorus), and the amount of less palatable substances such as lignin, or chemical inhibitors [23]. Environmental variables including water temperature, nutrient concentration or flow are extrinsic factors [24]. Plant organic matter decomposition is determined by the effect of interaction between the plant material quality and the environmental variables. Additionally, the decomposition velocity may be very important for biofilm development since slower decomposition enables the organic substrate to remain in the system longer with consequently, greater biofilm biomass development, as occurs in the wood [25].

Living plants. Biofilms growing on living plants are named epiphytic biofilms [17]. Submersed plants and macroalgae living in wetlands and littoral zones are a favorable habitat for microbial growth. Aquatic plants present vast potential for microbial colonization and are frequently covered by a dense growth of algae, bacteria and other organisms favoring biofilm formation [21]. However, in deeper parts of the plant, bacteria might be favored due to light reduction produced by the host plant, which affects algal development. Bacterial groups, Cytophaga-Flavobacteria-Bacteroidetes and Alpha-proteobacteria dominate epiphytic bacterial biofilms in marine and freshwater environments [26].

Plant and epiphytic biofilm interact in many ways, and the interaction may be both synergic and antagonistic. The biofilm provides macrophytes with organic compounds and carbon dioxide, and it also mediates nutrient uptake and enhances nutrient recycling [27]. In addition, some bacterial species produce compounds against biofouling organisms while some other species enhance plant growth [28]. In return, plants provide a substrate for biofilm formation and exude some gases such as methane and different organic compounds from the root zone, which are used by some biofilm bacteria. Consequently, different plant species, plant parts and environmental conditions may influence epiphytic biofilms, determining microbial community composition and densities [26].

Suspended aggregates. Suspended aggregates made of microorganisms, organic and inorganic particles are highly fragile structures suspended in fresh and seawater (usually after increased nutrients input) [29] and are usually named lake snow, river snow or marine snow.

Marine snow consists of aggregates of diatoms, filamentous cyanobacteria, bacteria, protozoans and diverse organisms with particles from the surrounding water, glued together in a polymeric matrix released from phytoplankton and bacteria. Anoxic conditions may exist within the snow particles so that diverse aerobic and 
anaerobic microbes colonize different niches [30]. Similar aggregates are formed in some water treatment systems, where they are called granules.

Aggregation is a complex process (involving microbes) and is controlled mainly by particle density, size and shape, settling velocity and the surface properties of the particle [31]. Furthermore, aggregates are affected by the degree of porosity and the flux of both water and nutrient to and from microbial cells on the surface.

Man-made surfaces. Diverse man-made surfaces including industrial and potable water system piping can support biofilm formation, where biofouling may occur by the deposition and growth of bacterial cells or flocs. In drinking water distribution systems AlphaBeta- and Gamma-proteobacteria and Gram-positive bacteria are more dominant bacterial groups [32]. In marine environments, man-made structures, such as oil and gas installations, aquaculture nets, and ship hulls, can also provide surfaces for attachment. In this sense, the attachment of diatoms along with bacteria on manmade structures constitutes a major problem for artificial structures immersed in the marine environment [32, 33].

The formation of attached microbial communities to man-made surfaces follows similar sequential steps to those observed for inert natural substrates (such as rock and sediments) [30, 33]. First, the surface is conditioned with a film of polysaccharides and proteins then, pioneer planktonic cells attach to the surface, followed the progressive arrival and biological adhesion of other microbes to form microcolonies until the mature biofilm develops.

\section{Species-to-Species Interactions in Biofilms}

Quorum sensing (QS) that also refers to as intercellular signaling is a regulatory mechanism that plays a very significant role in the regulation of bacterial biofilm formation [34]. In different environments, biofilms frequently exist as multispecies communities. The increase in the cell densities of such communities may result in high concentrations of QS signals in these communities. This interaction is very important and highly valuable for the microbial strains producing the signals and those occupying the same habitat [34]. In recent times, the importance of interspecies QS in shaping multispecies communities has been of high growing interest. Fig. 1 outlines some major determinants for QS in multispecies biofilms.

Synergistic interactions. In natural environments, multispecies biofilms exist as highly complex and dynamic entities. The presence of some microbial species in such

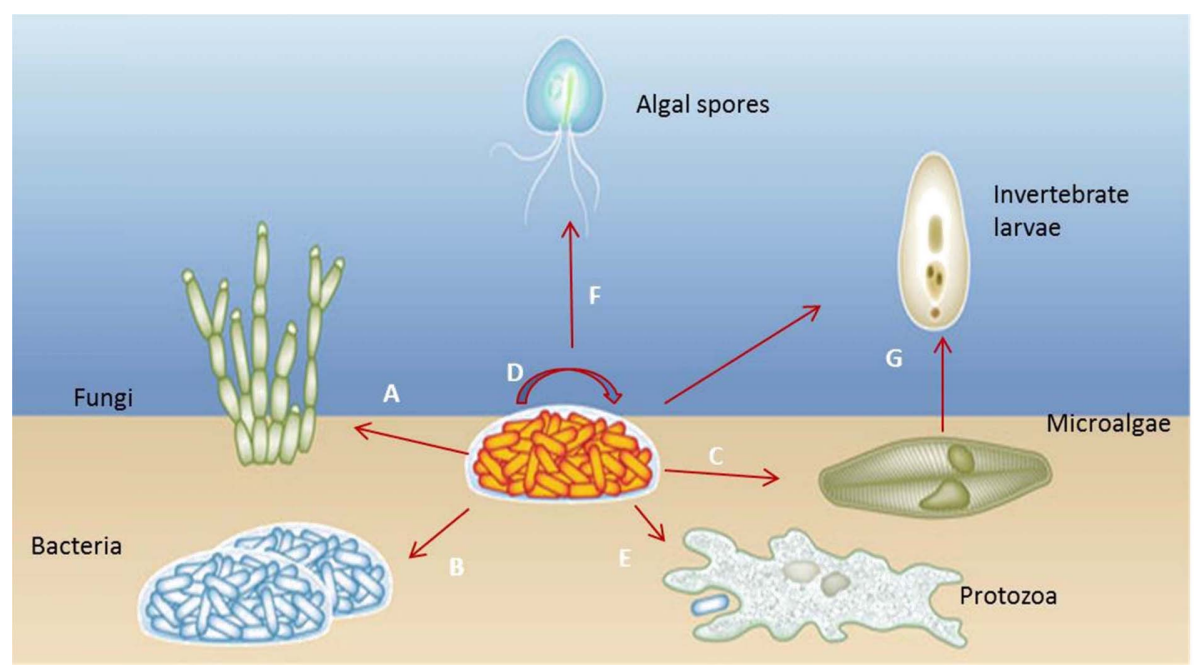

Fig. 1. Communication in a natural multispecies biofilm. Biofilm communities are shaped by various interactions between microbial species, including (1) competition between bacteria populations and their neighbours such as fungi (A), bacteria (B) and microalgae (C), (2) quorum sensing derived from clonal growth in microcolonies (D) which may induce the protection against protozoa (E) and (3) interactions with second colonizers such as macroalgae spores (F) and invertebrate larvae (G). 
a multispecies community positively promotes and improves inter and intra-species communications as well as the overall integrity of the formed biofilm. A typical example of synergistic interaction in the aquatic environment is the occurrence of Legionella pneumophila (which is often a minority member) in multispecies biofilm in freshwater and other natural environments [35]. Some bacterial species constituting the multispecies biofilm readily promotes $L$. pneumophila survival and persistence in such community [36]. Bacterial species interestingly reported to provide synergistic interactions to the long term and continuous L. pneumophila persistence in multispecies biofilm include Pseudomonas fluorescens, P. putida, Flavobacterium sp., Klebsiella pneumonia and Empedobacter breve [36]. These bacteria produce matrix materials (mainly capsular and extracellular) which promote adherence. Also, certain growth factors are provided by these bacterial species which consequently stimulate the growth and viability of $L$. pneumophila in such a multispecies biofilm community [36].

Furthermore, synergistic interactions are often augmented by the AI-2 QS in multispecies biofilms [37]. Dual bacterial species biofilms can also be formed even if only one of the bacterial species in the biofilm produces AI-2 signal. This is of immense interest, depicting species cooperation in multispecies biofilm communities. In the case of total inhibition and/or inactivation of AI-2 signals, mixed species biofilms become disrupted. In another instance, some microbial phenotypes become enhanced only in a multispecies biofilm [38]. Nevertheless, this majorly indicates the synergistic QS interactions which can only be unraveled via in-depth molecular studies.

Antagonistic interactions. Given the constant and fierce competition for nutrients within multispecies biofilms in a niche, certain mechanisms and products often regulate the survival of microbial species in such community. The most common mechanisms which augment competition within microbial species in biofilm are the production of bacteriocin (which is QS-regulated) and low $\mathrm{pH}$ [39]. $P$. aeruginosa, Sphingomonas sp., Burkholderia cepacia, Acidovorax sp. and Aeromonas hydrophila inhibit $L$. pneumophila once they exist in multispecies biofilm in freshwater or other natural environments [36]. Basically, homoserine lactone QS molecule of $P$. aeruginosa, as well as bacteriocins produced readily inhibit $L$. pneumophila [40]. An and colleagues [41] reported that in a dual-biofilm system between $P$. aeruginosa and Agrobacterium tumefacien, the former often dominate the later during the growth of such biofilm by utilizing QS. Also, with the use of a QS molecule known as farnesol, yeast species appear to take a direct approach in tackling other microbial competitors within the multispecies biofilm community. S. aureus shows enhanced susceptibility to antibiotic as well as decreasing the formation of biofilm when exposed to farnesol [42]. Furthermore, in multispecies biofilm between yeast and other species (especially bacteria), yeast outsmarts other members of the biofilm through the usage of farnesol, a QS molecule. The formation of biofilm decreases with increased susceptibility to antibiotics when $S$. aureus is exposed to farnesol [42].

It has also been reported that through QS, matured bacterial biofilms can prevent eukaryotic predation [43]. Another classical example is the $P$. aeruginosa degradation of farnesol, the QS signal which is optimally needed for the filamentation, maturation and biofilm formation by $C$. albicans. By utilizing virulence factors regulated by QS, P. aeruginosa can kill C. albicans [44].

Listening but not talking. Some microbial species cohabiting in a multispecies biofilm often respond to QS signals produced by other species within the biofilm, thereby altering their physiology. The virulence factors of $P$. aeruginosa become upregulated in the presence of AI-2, although $P$. aeruginosa does not produce AI-2. This phenomenon may be of importance in multispecies environments such as cystic fibrosis (CF) airway infections [45]. Similarly, bacteria in the ocean produce N-Acyl homoserine lactones (AHL) molecules which attract green seaweed's (Ulva) [46]. Bacterial biofilms colonizing surfaces often initiate biofouling, before the secondary colonization by algae and other eukaryotic organisms [47].

QS signaling interference. Studies conducted lately showed explicitly the capability of different organisms to degrade QS signals [34]. Once QS signals are degraded, their propagation within biofilm regions is prevented. The degradation of QS signal may prevent signal propa- 
gation between regions forming biofilm in microbial communities existing in a spatially structured system. This may further result in spatial confinement of the signal to different regions constituting the microbial community.

AHL-lactonases and AHL-acylases are quorum quenchers very common in AHL systems [48]. While the hydrolyzation of the lactone ring is conducted by AHLlactonases, AHL-acylases hydrolyze amide linkages. AHL-lactonases exist in several species of bacteria, although found foremost in Bacillus spp [49]. Only a few bacteria including $P$. aeruginosa and Ralstonia spp. are shown to produce AHL-acylases [50, 51].

The success achieved by microbial species in interfering QS signaling is through the use of QS signal mimics [52]. The inhibition of some strains of $S$. aureus QS by truncated peptides produced by some $S$. aureus agr QS systems is a clear example of QS interference [53].

Another instance of bacterial QS inactivation by eukaryotes was recently discovered in the Australian marine macroalga, Delisea pulchra, which disrupts $P$. aeruginosa AHL signaling. Thus, hindering biofilm formation on leaves surfaces by microorganisms [54].

Jamming of QS in multispecies biofilms. Although wide range of functions, including the control of virulence traits and bacterial biofilm are regulated by $\mathrm{QS}$, and An and others [41] noted that the development of mixed species populations is also actively carried out by QS. As earlier stated above, QS interference, inhibition and impairment can be carried out by individual bacterial species involved in biofilm. Also, QS molecules can be degraded by certain enzymes produced by bacteria [48]. The formation of biofilm by bacteria is directly affected by QS interferences within such community. A typical example is the inability of $V$. cholerae to form biofilm in the presence of AiiA, an AHL lactonase, produced by Bacillus cereus [55]. Also, the formation of biofilm by $P$. aeruginosa is often inhibited through interference with $P$. aeruginosa $\mathrm{PAO} 1 \mathrm{QS}$ in the presence of amines (aliphatic) and phenolic groups from other bacteria [56].

Bacterial communication signals and degrading enzymes can be found in the oral cavity. The colonization by $S$. mutans depends proportionately on their interactions with other early microbial species, such as
S. gordonii colonizing the dental system as lately described in two different studies. The success of $S$. mutans in biofilm formation in such a multispecies system is usually inactivated by serine protease challisin, which is secreted by $S$. gordonii, which interfere with a QS signals (competence- stimulating peptide) responsible for the formation of biofilm and subsequent colonization and plaque development [57]. Furthermore, of immense interest within the oral cavity is the successful colonization by $S$. mutans in the presence of Actinomyces naelundii (also an early colonizer), with protease activity [58]. Thus, jamming of QS may significantly control and influence waterborne pathogen biofilm formation, as well as disease outbreaks.

\section{Factors Contributing to the Dynamics of Biofilm Composition in Water Distribu- tion Systems}

In DWDS, majority of microorganisms (95\%) exist as biofilms [59]. The presence of biofilms attached to the water supplies pipe inner surface in the DWDS serve as a major source for the high microbial population. This is of great public health significance as it affects the quality and wholesomeness of drinking water. Despite the diversities of the conditions (nutritional and physicochemical) in DWDS, biofilms evolved mechanisms to successfully survive and flourish [60]. Increased water turbidity, odor, taste, reduction in water flow as a result of reduced pressure, corrosion and most importantly distribution of pathogens are the various problems associated with biofilms formed by multiple microbial species in DWDS. $E$. coli, Helicobacter pylori, Salmonella spp. and a host of other human pathogens and various microbial toxins detrimental to humans are often trapped by multispecies biofilms in DWDS [61]. The presence of different groups of microbes including viruses, bacteria algae, protozoans and bacteria forming a mixed microbial community in the form of biofilm is of grave concern. Attention should be given to such relationships, molecular mechanisms involved and products formed in order to elucidate and control is effects on DWDS.

Once a negligible number of bacteria succeeds in accessing the water distribution system, they multiply spontaneously especially when environmental and nutritional conditions become favorable [62]. Biofilms 
formed in DWDS are influenced by the factors which include physicochemical conditions of the water, microbial resistance to disinfection, availability of nutrients, and types and materials of piping systems [63].

Water source. The origin and source of water used in distribution systems have major influence on microbial communities. Several studies extensively reported the effects of water source on the composition microbial community [63]. Furthermore, it was also reported that a clear distinction in the diversities and profiles of microbial community exist among different DWDSs. With the application of multivariate analysis of surface and groundwater, bacterial communities can be clearly distinguished from in DWDS. Nevertheless, recent studies indicated that flowing water is the major source of bacteria in DWDS [62].

Ineffective disinfectants. The application of disinfectants during water treatment has a direct influence on both microbial population and their diversity [63]. Besides the fact that disinfectants eliminate microbes once administered appropriately, they also facilitate the formation of certain substances which are easily biodegradable by microbes. These biodegradable substances (usually organic) further enhance the formation of biofilms, as these microbes utilize them as their major energy source [65]. Although bacterial population can rapidly decline on the application of appropriate concentration of chlorine during water treatment, Gibbs and colleagues [64] observed bacterial regrowth as the chlorine concentration decreases. Some disinfectants potency rapidly fades out before the dead-end of distribution systems thereby favoring massive regrowth of microbial cells.

Bacterial resistance to disinfectants. Findings by different authors lately revealed the high number of bacterial multiplication and survival in culturable quantities in the presence of appropriate concentrations of disinfectants. The presence of these bacteria depicts their resistance to disinfectants [66]. Presently, research on the roots and causes of bacterial resistance to chlorine in the United Kingdom and parts of Asia have commenced in earnest.

Bacterial resistance to chlorine, been one of the most widely used disinfectants should not be a surprise. This is because most distribution systems only apply chlorine and these bacterial over the years have gradually evolved resistance mechanisms against its effect [64]. Biofilm attachment (which often protects microbial cells) on surfaces in distribution system, microbial cells aggregation, cell spore formation among others are also factors contributing to increased bacterial resistance to disinfectants [67].

Availability of nutrients. In the presence of nutrients microbial growth blossom and the biofilm formation become enhanced. Microbial stability on distribution systems is usually affected by the quantity of nutrients, most especially organic carbon which supports microbial regrowth [60]. With regards to the nature and type of disinfectant used, assimilable organic carbon (AOC) presence can favor rapid bacterial regrowth in DWDS. There is a significant relationship between bacterial ability to form biofilm and AOC uptake. Furthermore, once AOC becomes reduced in the distribution systems, bacterial ability to form biofilm declines [66].

Plumbing and piping materials. In DWDSs, metallic, plastic and cementitious pipes are generally used. The quality of water in distribution system is directly related to the type of material used in such a system [68]. Once a material is capable of supplying nutrients and enriching microbial growth, the formation of biofilm will be greatly facilitated. Most developed countries screens piping material to ascertain that they influence drinking water quality, most especially microbial growth enhancers. In DWDS, it has been reported that high bacterial densities $\left(10^{9}\right.$ bacteria $\left.\mathrm{cm}^{-2}\right)$ exist in biofilms within pipe surfaces [69]. It has also been documented that microbes deteriorate concrete piping in distribution systems [64]. Such deterioration is often complex and requires different microbial groups.

Temperature and season. Increase or decrease in temperature influences microbial growth in the DWDS [61]. Temperature varies alongside seasons, with both influence microbial growth hence biofilm formation in distribution systems. Whereas some authors reported seasonal differences in microbial community within the DWDS [70], others opined that microbial communities are not affected by the change in seasons [63], hence biofilm formation. 


\section{Contribution of Biofilms in Disease Trans- mission via Water Distribution Systems}

It has been earlier stated that the source of water directly determines the kind of microbial composition and interaction within water distribution systems. Biofilms formation congregates different groups of microbial species which enables them to interact freely [62]. Many pathogens including Aeromonas spp, Klebsiella spp, Pseudomonas spp, Enterobacter spp amongst others have reportedly been isolated from biofilms in distribution systems containing both disinfected and non-disinfected water [67]. Of immense public health interest is the increase in planktonic bacterial population as a result of regrowth from biofilms or raw water source. Although drinking water quality is microbiological assessed based on indicators (coliforms) presence, it is important that heterotrophic bacterial population should also be controlled as many have successfully become opportunistic pathogens causing serious infections in humans [71, 72].

Several emerging and re-emerging human pathogens such as Yersinia enterocolitica, Campylobacter spp, enterotoxigenic E. coli, Helicobacter pylori, Salmonella Typhimurium and also Cryptosporidium parvum have reportedly survived in biofilms in water distribution systems [73]. Multispecies biofilms involving cells of bacteria and cysts of protozoans, yeast cells and other species of fungi and algae can harbor and provide a favorable environment for the survival and proliferation of pathogens. These pathogens can subsequently detach into water flow in the distribution systems.

In most cases, the public health significance of biofilm is salient and indirect. It is glaring that biofilms can weaken the pipe integrity through corrosion and also compromise the use of coliforms as indicators. Aesthetic problems may not pose a direct health risk to the public but are clear indicators of biofilm presence and flaws in the system which may affect the public.

Role of biofilms in disease transmission and water quality. Bacterial adhesion to solid surfaces and their eventual formation of biofilms have a major impact on disease transmission and persistence [74]. Once biofilms are formed in the aquatic and other natural environments, different pathogens become residential as they are sheltered from adverse environmental factors capable of inhibiting or destroying them. This is also applicable to pathogens protected by biofilms in humans and other animals, which consequently evade the immune system of their host and also withstand the effects of antibiotics [73].

In recent times, large water bodies, especially oceans are increasingly been discovered to be major pathogen sources [75]. These water bodies accommodate different biofilm-forming pathogens which become integral members of such an environment. Fong and colleagues [73] gave an extensive description of the relationship existing between $V$. cholerae biofilm and cholera transmission and outbreaks. To fully unravel the role of biofilms in disease transmission, it is necessary to understand the molecular mechanisms of biofilm formation by bacteria.

Environmental biofilms produced by pathogens are capable of transmitting such pathogens to different communities, thereby initiating disease outbreaks [74]. A typical example of this phenomenon has been shown in Bangladesh, being an endemic area for cholera. Toxigenic $V$. cholerae were isolated from biofilms in surface water. Furthermore, sufficient quantity of $V$. cholerae cells existing as biofilms in cholera patients are capable of initiating infections once shedded [75-77]. As such, $V$. cholerae biofilms have vital role in both the survival and distribution of $V$. cholera. Most importantly, other pathogens associated with water are capable of exhibiting similar patterns in their ecology and also the epidemiology of diseases they cause.

The results of our previous study further indicated the capability of $V$. cholera to form biofilms on plexiglass discs, which are abiotic surfaces in Bangladesh aquatic environment. The biofilms were also observed to provide $V$. cholerae with a microenvironment in which rugose variants can persist [13].

Colonized with $V$. cholera in the form of biofilms particulates and planktons in the seawater of the Bay of Bengal further introduces cholera bacteria into the surrounding brackish ecosystems in Bangladesh. Major serogroups of V. cholera (including O1 and O139) living in biofilms have been isolated in all seasons throughout the year, responsible for the endemic nature of cholera in that entire region [18, 78]. Some serotypes of $V$. cholera exist in nonculturable state thrive far better in aquatic 
environment than when shed in stools of cholera patients [18], which are later washed back to the aquatic environment.

Protozoan grazing of aquatic biofilms: reservoirs of pathogenic bacteria. In a microbial food chain, bacteria within the natural environment become food for other living organisms including protozoa. This eventually depletes the number of bacteria within such habitats. Nevertheless, bacteria have developed diverse strategies against protozoa predation. The formation of biofilms is one of the most effective ways to avoid protozoan grazing. Also, bacteria develop other strategies against protozoan predation; various changes in the bacterial physiology and morphological changes like decreased biomass [79].

The ability of protozoa to fully colonize bacterial biofilms in the environment occurs in three stages. Heterotrophic flagellates are often the first to colonize surfaces immediately after exposure as a result of their mobility and high population, after which ciliates and amoebae. The success associated with grazing relies basically on the grazer's mode of nutrition as well as the bacterial biofilm developmental stage [77, 79]. Clearly, in contrast to planktonic cells, protozoa encounter a different feeding environment once biofilms are formed. As previously reported, there is 1.75 higher levels of biofilm metabolism when ciliate grazers are introduced to biofilms formed by the yeast-Cryptococcus spp when compared with non-grazing controls. The preference for the matrix of bacterial biofilms as against individual biofilm cells depicts EPS as nutrient source for protist. Studies have shown that the formation of microcolony and the production of QS induced inhibitors by bacterial biofilms are able to resist protozoan grazing [10] Fig. 2.

Apart from the above-stated mechanisms developed by bacterial biofilms against protozoan predation, it has been recently discovered that extensive biofilm formation arising as a result of the cooperation involving different species of bacteria can also resist predation [79]. Also, certain unknown toxin produced by $V$. cholera biofilms as induced by QS has been reported to greatly resist grazing most especially in fully matured biofilms [80]. In the aquatic environment, heterotrophic flagellates stimulate biofilm formation as individual bacterial cells greatly reduced [78]. Furthermore, it has been reported that protozoan non-selective grazing and release of nutrients cause stimulation which results in the overwhelming domination by fast-growing bacteria, thereby outgrowing their predators. The inability of mobility makes biofilms unable to escape protozoan grazing which unduly increases the intensity of grazing pressure. The abundance of bacterial biofilms in diverse environments is an indication of high anti-predator and increased fitness with immense mechanisms for grazing resistance long evolved as a result of close ecological associations between bacteria and protozoa.

Certain flagellates act as bacterivores in the aquatic environment, thereby affecting the spontaneous development of biofilm via sloughing and fragmentation. This further alters the distribution of EPS and other individual biochemical constituents of biofilms, reducing the chances of multispecies biofilm formation. Some protozoa such as Acanthamoeba castellanii and Colpoda maupasi
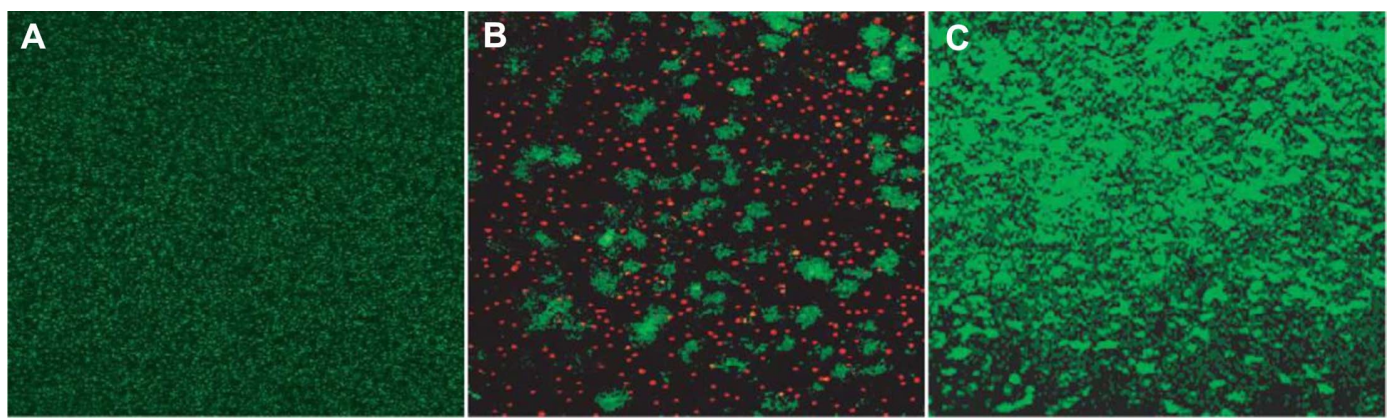

Fig. 2. The effects of flagellate Rhynchomonas nasuta grazing on Pseudomonas aeruginosa biofilms. (A) Three-day-old biofilms of $P$. aeruginosa PAO1 growing without flagellate. (B) Three-day-old biofilms of $P$. aeruginosa PAO1 growing with flagellate. (C) Seven-day-old biofilms of $P$. aeruginosa PAO1. Biofilms were regrown for 3 days before the addition of flagellate. Scale bar $=50 \mu \mathrm{m}$ $[14,150]$. 
thrive predominantly as biofilm grazer. They become integrated into biofilm thereby reducing the thickness of biofilm by up to $60 \%$ [70, 79]. Queck and others [43] showed that protozoan predation using different modes of feeding and motility gave rise to different structures of multispecies biofilms morphology. Of beneficial to bacteria is the increase mass transfer of nutrient into biofilms as a result of protozoan grazing, thus acceleration microbial growth [80].

Bacteria living in a biofilm can change their response to protozoa, thereby using such protozoa as pathogens reservoir. Amoebae harbor Listeria spp, Mycobacterium spp. and several other pathogens which can subsequently be released to the environment. Unique and more invasive forms of pathogens with biochemical and morphological changes result from protozoa involved in biofilm grazing than those cultured in vitro [80]. It is now obvious that protozoa within the environment involved fully in the distribution and maintenance of pathogens. More studies will further reveal the molecular mechanisms involved in protozoan grazing, biofilm resistance and pathogen persistence in the environment.

\section{Conclusive Remarks}

Aquatic microbial flora interacts within their environment to form multispecies biofilms. This complex interaction enables the survival and transmission of pathogens and thereby affects the water quality since large components of human health issues in developing countries are unsafe water, poor sanitation and inappropriate hygiene. Unlike the mono-species biofilms in clinical and laboratory settings, multispecies biofilms form dynamic microbial communities with extensive interspecies interactions in aquatic environment. Multispecies biofilms formation can be highly influenced by each member of such a mixed microbial community as well as the physicochemical parameters of the water. The biofilm-forming potentials of pathogens in DWDS and their interaction within a multispecies microbial community involving autochthonous aquatic bacteria which favors the persistence and dissemination of pathogens needed to be further explored. Future research should focus on the multispecies biofilms in aquatic environments to lessen the contamination from such settings. Since ecological interactions are major drivers for microbial community modeling, it becomes necessary to fully ascertain the mechanisms underlying microbial ecology of multispecies biofilms in the aquatic environment. Physiological and morphological heterogeneity coupled with both intrinsic and extrinsic interrelations make environmental biofilms much more complex entities. Further studies concentrating on the antagonistic interactions in multispecies biofilm in the aquatic environment will provide clues on improving water quality and safety. Furthermore, the use of promising molecular technologies will fully unravel the detailed interactions between biofilms in aquatic environment and the operating ecological processes, thus aiding in managing multispecies biofilms to provide services to society.

\section{Acknowledgments}

This research has been financed by the Bangladesh Academy of Science under BAS-USDA program code no. LSC 33

\section{Conflict of Interest}

The authors have no financial conflicts of interest to declare.

\section{References}

1. World Health Organization (WHO) 2010. Millennium Development Goals: Progress towards the Health-Related Millennium Development Goals; World Health Organization (WHO): Geneva, Switzerland. Available online: http://www.who.int/topics/millennium_development_goals/en/

2. World Health Organization (WHO) 2015. Water Sanitation and Health. Available online: http://www.who.int/water_sanitation_ health/diseases

3. Alhamlan FS, Al-Qahtani AA, Al-Ahdal MN. 2015. Recommended advanced techniques for waterborne pathogen detection in developing countries. J. Infect. Dev. Con. 9: 128-135.

4. Justin T, Cristal Z, Karsten Z. 2015. Unraveling interactions in microbial communities - from co-cultures to microbiomes. J. Microbiol. 53: 295-305.

5. Hall-Stoodley L, Costerton JW, Stoodley P. 2004. Bacterial biofilms: from the natural environment to infectious diseases. Nat. Rev. Microbiol. 2: 95-108.

6. Donlan RM, Costerton JW. 2002. Biofilms: survival mechanisms of clinically relevant microorganisms. Clin. Microbiol. Rev. 15: 167-193.

7. Jahid IK, Ha SD. 2012. A review of microbial biofilms of produce: future challenge to food safety. Food Sci. Biotechnol. 21: 299-316.

8. Davey ME, O'toole GA. 2000. Microbial biofilms: from ecology to molecular genetics. Microbiol. Mol. Biol. Rev. 64: 847-867.

9. McKew BA, Taylor TJ, Underwood UJ. 2010. Resistance and resil- 
ience of benthic biofilm communities from a temperate saltmarsh to desiccation and rewetting. ISME J. 5: 30-41.

10. Matz C, Kjelleberg S. 2005. Off the hook-how bacteria survive protozoan grazing. Trends Microbiol. 13: 302-307.

11. September S, Els F, Venter S, Brozel V. 2007. Prevalence of bacterial pathogens in biofilms of drinking water distribution systems. J. Water Health. 5: 219-227.

12. Kim JG, Park SJ, Quan ZX, Jung MY, Cha IT, Kim SJ. 2014. Unveiling abundance and distribution of planktonic Bacteria and Archaea in a polynya in amundsen sea, Antarctica. Environ. Microbiol. 16: 1566-1578.

13. Islam SM, Jahid IK, Rahmn MM, Rahman ZM, Islam SM, Kabir SM, 2007. Biofilm acts as a microenvironment for plankton-associated Vibrio cholerae in aquatic environment of Bangladesh. Microbiol. Immunol. 51: 369-379.

14. Johnson L. 2007. Microcolony and biofilm formation as a survival strategy for bacteria. J. Theor. Biol. 251: 24-34.

15. Romani AM, Giorgi A, Acuna V, Sabater S. 2004. The influence of substratum type and nutrient supply on biofilm organic matter utilization in streams. Limnol. Oceanog. 49: 1713-1721.

16. Terlizzi A, Faimali M. 2010. Fouling on artificial substrata. In Biofouling, Durr S and Thomason JC. eds. pp. 170-179. (Blackwell Publishing Ltd, Oxford, UK).

17. Vadeboncoeur $Y$, Steinman AD. 2002. Periphyton function in lake ecosystems. Sci. W. J. 2: 1449-1468.

18. Graba M, Sauvage S, Moulin FY, Urrea G, Sabater S, SanchezPerez JM. 2013. Interaction between local hydrodynamics and algal community in epilithic biofilm. Water Res. 47: 2153-2163.

19. Murdock JN, Dodds WK. 2007. Linking benthic algal biomass to stream substratum topography. J. Phycol. 43: 449-460.

20. Maclntyre HL, Geider RJ, Miller DC. 1996. Microphytobenthos: the ecological role of the 'Secret Garden' of unvegetated, shallowwater marine habitats. I. Distribution, abundance and primary production. Estuaries 19: 186-201.

21. Boulton AJ, Findlay S, Marmonier P, Stanley EH, Valett HM. 1998. The functional significance of the hyporheic zone in streams and rivers. Ann. Rev. Ecol. Evol. Syst. 29: 59-81.

22. Danger M, Cornut J, Chauvet E, Chavez P, Elger A, Lecerf A. 2013. Benthic algae stimulate leaf litter decomposition in detritusbased headwater streams: a case of aquatic priming effect? Ecology 94: 1604-1613.

23. Cornwell WK, Cornelissen JHC, Amatangelo K, Dorrepaal E, Eviner VT, Godoy O. 2008. Plant species traits are the predominant control on litter decomposition rates within biomes worldwide. Ecol. Lett. 11: 1065-1071.

24. Tank JL, Rosi-marshall EJ, Griffiths NA, Entrekin SA, Stephen ML. 2010. A review of allochthonous organic matter dynamics and metabolism in streams. Freshwater Sci. 29: 118-146.

25. Hempel M, Blume M, Blindow I, Gross EM. 2008. Epiphytic bacterial community composition on two common submerged macrophytes in brackish water and freshwater. BMC Microbiol. 8: 58.

26. Rao D, Webb JS, Kjelleberg S. 2006. Microbial colonization and competition on the marine alga Ulva australis. Appl. Environ.
Microbiol. 72: 5547-5555.

27. De Beer D, Stoodley P. 2006. Microbial biofilms. In: The Prokaryotes. In Dworkin, M., Falkow, S, Rosenberg E, Schleifer KH, Stackebrandt E, eds. pp. 904-937. Springer, New York, NY, USA.

28. Alldredge A. 1998. The carbon, nitrogen and mass content of marine snow as a function of aggregate size. Deep-Sea Res. Part 1 45: 529-541.

29. Simon M, Grossart H, Schweitzer B, Ploug H. 2002. Microbial ecology of organic aggregates in aquatic ecosystems. Aqua. Microbial. Ecol. 28: 175-211.

30. Tokajian ST, Hashwa FA, Hancock IC, Zalloua PA. 2005. Phylogenetic assessment of heterotrophic bacteria from a water distribution system using 165 rDNA sequencing. Can. J. Microbiol. 51: 325-335.

31. Salta M, Wharton JA, Blache Y, Stokes KR, Briand JF. 2013. Marine biofilms on artificial surfaces: structure and dynamics. Environ. Microbiol. 15: 2879-2893.

32. Gaudes A, Artigas J, Romani AM, Sabater S, Munoz I. 2009. Contribution of microbial and invertebrate communities to leaf litter colonization in a Mediterranean stream. J. N. Am. Benthol. Soc. 28: 34-43.

33. Burke C, Thomas T, Lewis M, Steinberg P, Kjelleberg S. 2011. Composition, uniqueness and variability of the epiphytic bacterial community of the green alga Ulva australis. ISME J. 5: 590-600.

34. Irie Y, Parsek MR. 2008. Quorum sensing and microbial biofilms. Curr. Top. Microbiol. Immunol. 322: 67-79.

35. Declerck P. 2010. Biofilms: the environmental playground of Legionella pneumophila. Environ. Microbiol. 12: 557-566.

36. Stewart CR, Muthye V, Cianciotto NP. 2012. Legionella pneumophila persists within biofilms formed by Klebsiella pneumoniae, Flavobacterium sp., and Pseudomonas fluorescens under dynamic flow conditions. PLoS One. 7: e50560.

37. Kolenbrander PE, Andersen RN, Blehert DS, Egland PG, Foster JS, Palmer RJ. 2002. Communication among oral bacteria. Microbiol. Mol. Biol. Rev. 66: 486-505.

38. Burmølle M, Webb JS, Rao D, Hansen LM, Sørensen SJ, Kjelleberg S. 2006. Enhanced biofilm formation and increased resistance to antimicrobial agents and bacterial invasion are caused by synergistic interactions in multispecies biofilms. Appl. Environ. Microbiol. 72: 3916-3923.

39. Tait K, Sutherland I. 2002. Antagonistic interactions amongst bacteriocin-producing enteric bacteria in dual species biofilms. J. Appl. Microbiol. 93: 345-352.

40. Mallegol J, Duncan C, Prashar A, So J, Low DE, Terebeznik M. 2012. Essential roles and regulation of the Legionella pneumophila collagen-like adhesin during biofilm formation. PLoS One. 7: e46462.

41. An D, Danhorn T, Fuqua C, Parsek MR. 2006. Quorum sensing and motility mediate interactions between Pseudomonas aeruginosa and Agrobacterium tumefaciens in biofilm cocultures. Proc. Natl. Acad. Sci. USA 103: 3828-3833.

42. Jabra-Rizk MA, Meiller TF, James CE, Shirtliff ME. 2006. Effect of farnesol on Staphylococcus aureus biofilm formation and antimicrobial susceptibility. Antimicrob. Agents Chemo. 50: 1463-1469. 
43. Queck SY, Weitere M, Moreno AM, Rice SA, Kjelleberg S. 2006. The role of quorum sensing mediated developmental traits in the resistance of Serratia marcescens biofilms against protozoan grazing. Environ. Microbiol. 8: 1017-1025.

44. Duan K, Dammel C, Stein J, Rabin H, Surette HG. 2003. Modulation of Pseudomonas aeruginosa gene expression by host microflora through interspecies communication. Mol. Microbiol. 50: 1477-1491.

45. Tait K, Joint I, Daykin M, Milton DL, Williams P, Cámara M. 2005. Disruption of quorum sensing in seawater abolishes attraction of zoospores of the green alga Ulva to bacterial biofilms. Environ. Microbiol. 7: 229-240.

46. Dong YH, Zhang LH. 2005. Quorum sensing and quorum quenching enzymes. J. Microbiol. 43: 101-109.

47. Dong YH, Xu JL, Li XZ, Zhang LH. 2000. AiiA, an enzyme that inactivates the acylhomoserine lactone quorum-sensing signal and attenuates the virulence of Erwinia carotovora. Proc. Natl. Acad. Sci. USA. 97: 3526-3531

48. Huang JJ, Han Jl, Zhang LH, Leadbetter JR. 2003. Utilization of acyl-homoserine lactone quorum signals for growth by a soil pseudomonad and Pseudomonas aeruginosa PAO1. Appl. Environ. Microbiol. 69: 5941-5949.

49. McDougald D, Rice SA, Kjelleberg S. 2006. Bacterial quorum sensing and interference by naturally occurring biomimics. Ana. Bioana. Chem. 387: 445-453.

50. Chun CK, Ozer EA, Welsh MJ, Zabner J, Greenberg EP. 2004. Inactivation of a Pseudomonas aeruginosa quorum-sensing signal by human airway epithelia. Proc. Natl. Acad. Sci. USA 101: 35873590.

51. Hentzer M, Riedel K, Rasmussen TB. 2002. Inhibition of quorum sensing in Pseudomonas aeruginosa biofilm bacteria by a halogenated furanone compound. Microbiology 148: 87-102.

52. Augustine N, Kumar P, Thomas S. 2010. Inhibition of Vibrio cholerae biofilm by AiiA enzyme produced from Bacillus spp. Arch. Microbiol. 192: 1019-1022.

53. Musthafa KS, Saroja V, Pandian SK, Ravi AV. 2011. Antipathogenic potential of marine Bacillus sp. SS4 on N-acylhomoserine- lactonemediated virulence factors production in Pseudomonas aeruginosa (PAO1). J. Biosci. 36: 55-67.

54. Senadheera D, Cvitkovitch DG. 2008. Quorum sensing and biofilm formation by Streptococcus mutans. Adv. Exp. Med. Biol. 631: 178-188.

55. Wang BY, Deutch A, Hong J, Kuramitsu HK. 2011. Proteases of an early colonizer can hinder Streptococcus mutans colonization in vitro. J. Dent. Res. 90: 501-505.

56. Wingender J, Flemming HC. 2004. Contamination potential of drinking water distribution network biofilms. Water Sci. Technol. 49: 277-286.

57. Szewzyk U, Szewzyk R, Manz W, Schleifer KH. 2000. Microbiological safety of drinking water. Ann. Rev. Microbiol. 54: 81-127.

58. Armon R, Starosvetzky J, Arbel T, Green M. 1996. Survival of Legionella pneumophila and Salmonella typhimurium in biofilm systems. Water Sci. Technol. 35: 293-300.
59. Momba MNB, Kfir R, Venter SN, Cloete RE. 2000. An overview of biofilm formation in distribution systems and its impact on the deterioration of water quality. Water SA. 26: 59-66.

60. Eichler S, Christen R, Holtje C, Westphal P, Botel J, Brettar A. 2006. Composition and dynamics of bacterial communities of a drinking water supply system as assessed by RNA- and DNA-based $16 \mathrm{~S}$ rRNA gene fingerprinting. Appl. Environ. Microbiol. 72: 18581872.

61. Ling F, Hwang C, LeChevallier MW, Andersen GL, Liu WT. 2016. Core-satellite populations and seasonality of water meter biofilms in a metropolitan drinking water distribution system. ISME J. 10: 582-595.

62. Roeselers G, Coolen J, van der Wielen PW, Jaspers MC, Atsma A, de Graaf B. 2015. Microbial biogeography of drinking water: patterns in phylogenetic diversity across space and time. Environ. Microbiol. 17: 2505-2514.

63. Douterelo I, Jackson M, Solomon C, Boxall J. 2016. Microbial analysis of in situ biofilm formation in drinking water distribution systems: implications for monitoring and control of drinking water quality. Appl. Microbiol. Biotechnol. 100: 3301-3311.

64. Gibbs RA, Sutt JE, Croll J. 1990. Microbiological and trihalomethane responses to booster chlorination. J. IWEM 4: 131-139.

65. Olivieri VP, Bakalian AE, Bossung KW, Lowther ED. 1985. Recurrent coliforms in water distribution systems in the presence of free residual chlorine. pp. 651-666. In Jolley RL, Bull RJ, Davis WP, Katz S, Roberts MH (Jr) and Jacobs VAO (eds.) Water Chlorination: Chemistry, Environmental Impac t and Health Effects. Lewis Publishers, Inc., Chelsea, MI.

66. Camper MB, Ellis B, Buterfield P, Abernathy C. 1999. Development and structure of drinking water biofilms and techniques of their study. J. Appl. Microbiol. Symp. Suppl. 85: 1S-12S.

67. Escobar IC, Randall AA, Taylor JS. 2001. Bacterial growth in distribution systems: effect of assimilable organic carbon and biodegradable dissolved organic carbon. Environ. Sci. Technol. 35: 3442-3447.

68. Rogers J, Dowsett AB, Dennis PJ, Lee JV, Keevil CW. 1994. Influence of plumbing materials on biofilm formation and growth of Legionella pneumophila in potable water systems. Appl. Environ. Microbiol. 18: 32-51.

69. Olson BH. 1982. Assessment and implication of bacterial regrowth in water distribution systems. EPA. 600/52-82-072 U.S. Environmental Protection Agency.

70. Pinto AJ, Schroeder J, Lunn M, Sloan W, Raskin L. 2014. Spatialtemporal survey and occupancy-abundance modeling to predict bacterial community dynamics in the drinking water microbiome. MBio. 5: e01135-01114.

71. Mackay WG, Gribbon LT, Barer MR, Reid DC. 1998. Biofilms in drinking water systems - A possible reservoir for Helicobacter pylory. Water Sci. Technol. 38: 181-185.

72. Huq A, Whitehouse CA, Grim CJ, Alam M, Colwell RR. 2008. Biofilms in water, its role and impact in human disease transmission. Curr. Opin. Biotechnol. 19: 244-247.

73. Fong J, Karplus K, Schoolnik G, Yildiz F. 2006. Identification and 
characterization of $\mathrm{RbmA}$, a novel protein required for the development of rugose colony morphology and biofilm structure in Vibrio cholerae. J. Bacteriol. 188: 1049-1059.

74. Faruque S, Biswas K, Udden S, Ahmad Q, Sack D, Nair G, et al. 2006. Transmissibility of cholera: in vivo-formed biofilms and their relationship to infectivity and persistence in the environment. Proc. Natl. Acad. Sci. USA 103: 6350-6355.

75. Erken M, Farrenschon N, Speckmann S, Arndt H, Weitere M. 2012. Quantification of individual flagellate-bacteria interactions within semi-natural biofilms. Protist 163: 632-642.

76. Friman VP, Diggle SP, Buckling A. 2013. Protist predation can favour cooperation within bacterial species. Biol. Lett. 9: 20130548.

77. Sun S, Kjelleberg S, McDougald D. 2013. Relative contributions of Vibrio polysaccharide and quorum sensing to the resistance of Vibrio cholerae to predation by heterotrophic protists. PLoS One. 8: e56338.

78. Wey JK, Scherwass A, Norf H, Arndt H, Weitere M. 2008. Effects of protozoan grazing within river biofilms under semi-natural conditions. Aquat. Microb. Ecol. 52: 283-296.

79. Huws S, McBain A, Gilbert P. 2005. Protozoan grazing and its impact upon population dynamics in biofilm communities. J. Appl. Microbiol. 98: 238-244.

80. Dopheide A, Lear G, Stott R, Lewis G. 2011. Preferential feeding by the ciliates Chilodonella and Tetrahymena spp. and effects of these protozoa on bacterial biofilm structure and composition. Appl. Environ. Microbiol. 77: 4564-4572. 